\title{
Mathematic Analysis of Unbalanced Radial Distribution Systems with Distributed Generations
}

\author{
Saad OUALI ${ }^{1, *}$, Abdeljabbar CHERKAOUI ${ }^{2}$ \\ ${ }^{1,2}$ Laboratory of Innovative Technologies (LTI), National School of Applied Sciences, Abdelma lek Esaadi University, Tangier Morocco
}

How to cite this paper: OUALI, S., CHERKAOUI, A. (2018) Mathematic Analysis of Unbalanced Radial Distribution Systems with Distributed Generations. Journal of Applied Mathematics and Computation, 2(12), 543-556.

DOI: $10.26855 /$ jamc.2018.12.002

*Corresponding author: Saad OUALI, Laboratory of Innovative Technologies (LTI), National School of Applied Sciences, Abdelmalek Esaadi University, Tangier Morocco.

Email: saad.ouali1@gmail.com

\begin{abstract}
Changing in a system parameters produces an effect on the system performance. However, the impact of system parameters are not equal, some parameters have important influence while others may have less significant influence. In electrical distribution systems, the introduction of distributed generation, from renewable energy sources, had negatively influences their voltage plan. The active and reactive power produced and consumed by those distributed generations had change the ordinary operation of the classical radial distribution networks. In this paper a mathematical method is proposed to quantify the impact of the variation of active and reactive power on the voltage plan of radial distribution networks. The main aim of this paper is to understand how the voltage of each part of the network is influenced by the active and reactive power of distributed generation. The results obtained may be very useful to establish a control law to control the voltage plan, and to optimize the location of distributed generation and actuator, A case study is presented, with an application of the proposed method on a radial distribution system, the results presents the influence of voltage plan with respect to the variation of active and reactive power generated by distribution generations, and network characteristics.
\end{abstract}

Keywords

Mathematic analysis; Unbalanced three phase system; Radial distribution system; Power flow summation; Distributed generation; Voltage control; renewable energy.

\section{Introduction.}

DG affects classical radial distribution systems. The influence may manifest them either positively or negatively depending on the distribution system operating conditions and the DG characteristics. Positive impacts are generally called 'system support benefits', and include diversification of energy resources, reduction of on-peak operating cost, deferral of network upgrades, lower losses and lower transmission and distribution costs, and potential increase of service quality to the end-customer. Energy cost of renewable-based distributed generation, such as; hydro, wind, solar, geothermal, biomass and ocean energy, which are naturally scattered, when compared to the conventional generating plants is generally high because the social and environmental benefits could not be included in the cost account.

Distributed generation is known by various names like decentralized generation, dispersed generation, and embedded generation or redistributed energy. Any distributed generation creates electricity from many small energy sources, connected directly to the distribution network.

The amount of distributed generation such as photovoltaic solar and wind generations, being connected to the distribution systems is increasing, as result of their many technical, economic and environmental benefits. However, the degra- 
dation on the voltage plan, caused by the active and reactive power consumed and injected by those generations units, is reported as one of the main obstacles for the interconnection of a large amounts renewable units to the existing radial networks [1].

Unlike transmission systems, medium and low voltage distribution systems have high R/X ratio, strongly radial structure with large number of nodes, branches, and complex topology configurations that can be changed for maintenance activities, emergency operations or network configurations [2], which results in high power losses and may had to voltage instability, study have indicated that $13 \%$ of total power generation is wasted in line losses at distribution level.

Medium and low voltage distribution systems are designed and calibrated for a passif use, with unidirectional power flow from the MV/LV substation for the low voltage systems, or from the HV/MV substation in medium voltage systems to the end of the line. The penetration of distributed generators in distribution systems will challenge the correct functioning of those systems. Unfortunately, the classical models designed for high voltage network analysis, such as Newton-Raphson, Gauss-Seidel or Fast-Decoupled power flow is not adequate to distribution network.

References [2][3] report a comparative study of the most used algorithms, the power summation methods seems to be the best choice, thanks to its robustness and convergence properties.

A voltage control strategy is a challenging task due to the non-linear relationship between the active/reactive power and voltage magnitude. The implantation of such control will avoid disconnection of distributed production units and will increase the active power production [4].

In this paper, an analysis method is presented. The results obtained from the proposed method is useful in voltage control, loss reduction, network reinforcement, production planning, optimal location of distributed generation, reactive sources and voltage control actuator, and any applications which is necessary to predict the changes in voltage plan.

The main aim of this paper is to conceive a tool able to analysis unbalanced distribution networks. This tool can be used directly as a decision support tool for utilities in cases of recording a new distributed generation unit, or network extension, or as a calculation basis for an automatic voltage control.

In what follows, we present the different types of distributed generation, in section III, a modeling of unbalanced radial networks and its equipment is developed in section III, next in section IV the relationship between voltage magnitude, feeder characteristics and active/reactive power, able to mitigate the impact of distributed generation on network voltage is presented. In Section IV we provide the theoretical foundation of the proposed mathematic analysis method, and in section $\mathrm{V}$ we present a case study of the proposed method with the real unbalance distribution system, in section VI we discuss the results obtained from the case study.

\section{Types of Distributed Generation}

Several types of distributed generation can be distinguished, according to the technology, type of generators used, and depending on which primary energy they are exploiting, Fig 1 descript the existing types of distributed generations [7].

Generally 2 principals types of distributed generation can be differentiate:

- Conventional or traditional generators based on combustion engines

- Non-conventional generator as: Storage devices, electrochemical devices and renewable device.

\subsection{Traditional generators:}

Developed from automotive, truck turbo-charges and auxiliary power units, micro-turbines are small capacity combustion turbines, which can operate using fuel, sour gases, natural gas and propane, with outputs of $25 \mathrm{KW}$ to $500 \mathrm{KW}$ [8], while conventional gas turbine sizes range from $500 \mathrm{~kW}$ to 250 megawatts (MW). Micro-turbine generators can be divided into two general types: Recuperated or combined cycle and Unrecuperated or simple cycle, depending on the compressed air, if it is mixed with fuel and burned under constant pressure conditions, or it's boosting by the temperature recuperate from the exchanged heat. Due to its small size, relatively low investment costs, and low expected operation and maintenance costs, micro-turbines are expected to play an important role in the future medium and low voltage distribution networks. 


\subsection{Non-Traditional generators:}

Three types of non-conventional can be separated: Electrochemical devices, storage devices and renewable devices.

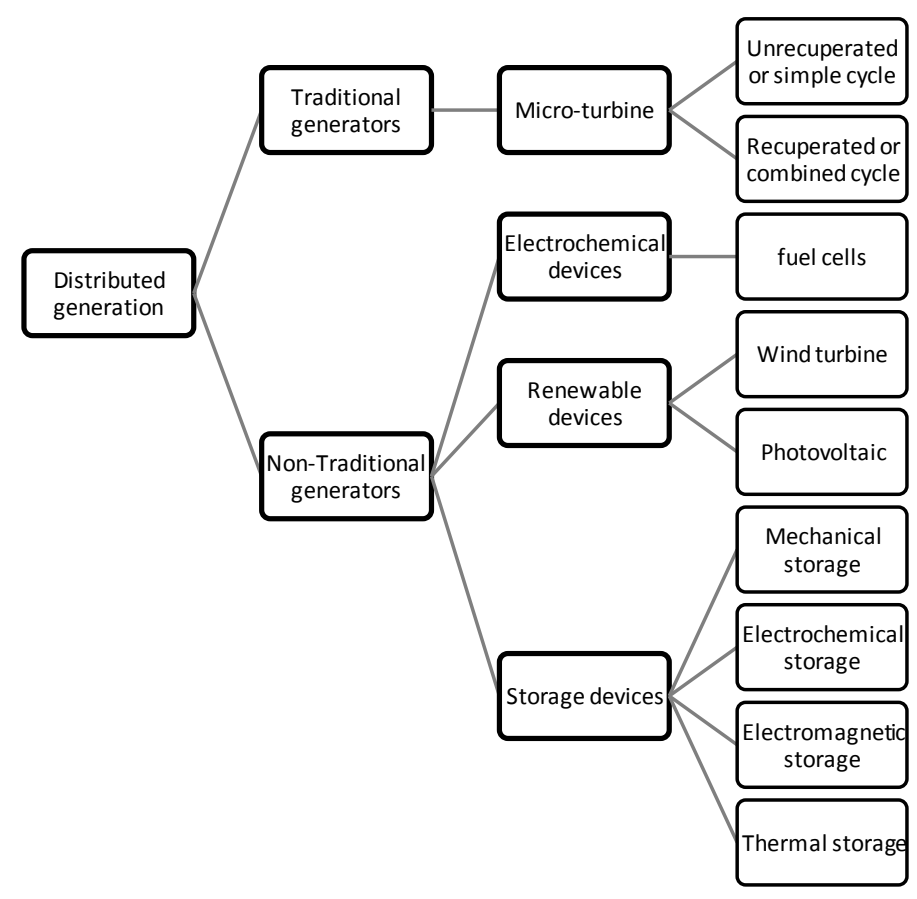

Figure 1. Types of distributed generation

\section{A. Electrochemical Devices:}

Electrochemical devices or Fuel cell are theoretically composed of an electrolyte in contact with an anode (negative electrode) and a cathode (positive electrode), in which the chemical energy is directly converted into electrical energy through electrochemical processes.

\section{B. Storage devices:}

Several types of storage devices are existing and using in electrical systems:

- Mechanical: hydroelectric dam, pumped energy transfer station - STEP, and flywheels.

- Electrochemical: batteries, batteries, hydrogen vector.

- Electromagnetic: superconducting coils, supercapacitors.

- Thermal: Sensible heat.

Storage devices are generally charges during low load demand and used with the need.

\section{Renewable devices:}

Several categories of renewable devices, classified according to the primary energy they exploit, are present on the network, such as: hydro, wind, solar, geothermal, biomass and ocean energy, which are naturally scattered. The renewable devices commonly used as distributed generation are photovoltaic and wind turbines. 


\section{Distribution System Modeling}

In North Africa, Medium voltage distribution networks consist of a three phase balanced system. However, the Low Voltage distribution networks are a three-phase four wires ( 3 phases and one neutral) unbalanced system. Both Low and Medium systems are composed of feeders, loads, and future distributed generation.

\subsection{Feeders Modeling:}

Generally, the length of most distribution line sections is short $(<80 \mathrm{~km})$ [9], the $\pi$ model is not used because line shunt capacitance is found to be negligible, the short line model is the model used for modelling feeders in distribution networks, as series impedance $(\mathrm{Z}=\mathrm{r}+\mathrm{y})$.

The voltage at the busbar "a" and a busbar a', are related by:

$$
V_{a \prime}^{a b c}=V_{a}^{a b c}-I_{a a^{\prime}}^{a b c} Z_{a a^{\prime}}^{a b c}
$$

Where $\mathrm{Z}_{\mathrm{aa}}$, is the impedance matrix of the feeder aa':

$$
Z_{a a^{\prime}}^{a b c}=\left[\begin{array}{ccc}
Z_{a a^{\prime}}^{a a} & Z_{a a^{\prime}}^{a b} & Z_{a a^{\prime}}^{a c} \\
Z_{a a^{\prime}}^{b a} & Z_{a a^{\prime}}^{b b} & Z_{a a^{\prime}}^{b c} \\
Z_{a a^{\prime}}^{c a} & Z_{a a^{\prime}}^{c b} & Z_{a a^{\prime}}^{c c}
\end{array}\right]
$$

In low voltage networks with three-phase four wires ( 3 phases and one neutral) unbalanced system, is shown in fig 2.

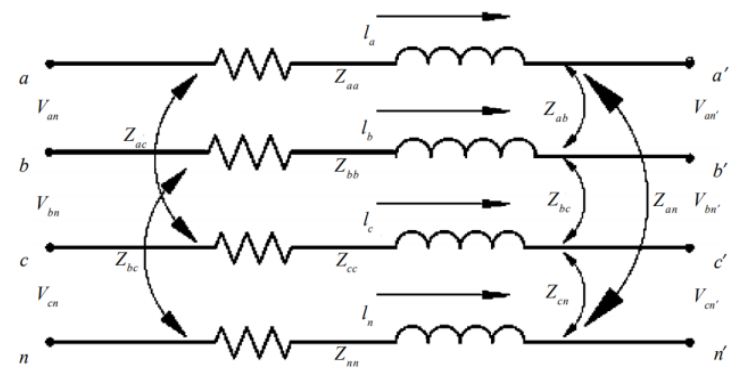

Figure 2. Low Voltage distribution system feeders model

The impedance matrix is represented are shown in equ (3),

$$
Z_{a a^{\prime}}^{a b c}=\left[\begin{array}{cccc}
Z_{a a^{\prime}}^{a a} & Z_{a a^{\prime}}^{a b} & Z_{a a^{\prime}}^{a c} & Z_{a a^{\prime}}^{a n} \\
Z_{a a^{\prime}}^{b a} & Z_{a a^{\prime}}^{b b} & Z_{a a^{\prime}}^{b c} & Z_{a a^{\prime}}^{b n} \\
Z_{a a^{\prime}}^{c a} & Z_{a a^{\prime}}^{c b} & Z_{a a^{\prime}}^{c c} & Z_{a a^{\prime}}^{c n} \\
Z_{a a^{\prime}}^{n a} & Z_{a a^{\prime}}^{n b} & Z_{a a^{\prime}}^{n c,} & Z_{a a^{\prime}}^{n n}
\end{array}\right]
$$

\subsection{Load Modeling:}

The load is generally modeling by constant power, constant current, constant impedance and composite loads. In static analysis, it is considered that the frequency deviation is insignificant and, only active and reactive load power can affect the voltage plan [9][10], The variation of the real and reactive power of a load with respect to the voltage magnitude is given in Equations (4) and (5),

$$
\begin{aligned}
& P=P_{0}\left(a_{0}+a_{1} V+a_{2} V^{2}+a_{3} V^{K 1}\right) \\
& Q=Q_{0}\left(b_{0}+b_{1} V+b_{2} V^{2}+b_{3} V^{K 2}\right)
\end{aligned}
$$


Where:

$$
\left\{\begin{array}{l}
a_{0}+a_{1}+a_{2}+a_{3}=1 \\
b_{0}+b_{1}+b_{2}+b_{3}=1
\end{array}\right.
$$

And:

$$
\left\{\begin{array}{c}
\mathrm{k} 1=\mathrm{k} 2=0 \text { for constant power loads } \\
\mathrm{k} 1=\mathrm{k} 2=1 \text { for constant current loads } \\
\mathrm{k} 1=\mathrm{k} 2=2 \text { for constant impedance loads } \\
\mathrm{k} 1=1.38, \mathrm{k}=3.22 \text { for exponential loads }
\end{array}\right.
$$

In Medium voltage distribution systems, the loads used are Shunt capacitors and transformers.

\subsection{DG modeling:}

Three principal kind of distributed generation model can be separate:

- DG can only produce active power without exchanging any reactive power; in this case, a DG is presented as a negative load model with zero reactive power.

- DG able to produce active power, but consumes reactive power, in this case, DG is modeling by the equations derived from the equivalent circuit of its induction generator.

- DG able to produce active power, and can regulate theirs reactive power, produce or consume reactive power, in case DG is modeling as the previous case, but the reactive power is setting constant.

\section{Steady State Voltage Plan and Distributed Generation}

The electrical network is shown in fig3.

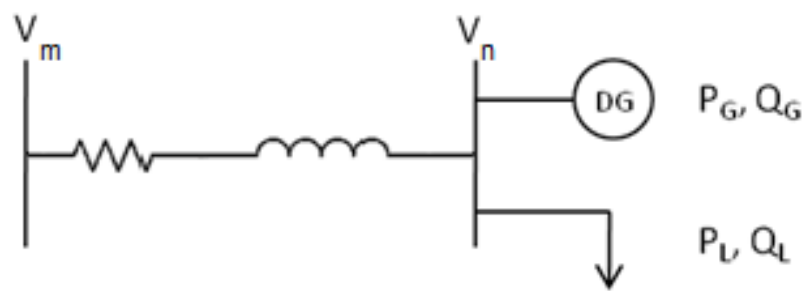

Figure 3. Electrical system with DG

The power between the node " $m$ " and " $n$ " is:

$$
S_{m}=P_{m}+j Q_{m}=\left(P_{m}+j Q_{m}\right)-\left(P_{m}+j Q_{m}\right)
$$

It can also be expressed as:

$$
S_{m}=V_{m} \cdot I_{m n}^{*}
$$

The current $\mathrm{I}_{\mathrm{mn}}$ between the nodes " $\mathrm{m}$ " and " $\mathrm{n}$ ":

$$
I_{m n}=\frac{P_{m}-j \cdot Q_{m}}{V_{m}^{*}}
$$

The relationship between the voltage of node " $m$ " can be obtained from equ (1) and (3) :

$$
\begin{gathered}
V_{m}=V_{n}+Z \cdot I_{m n}=V_{n}+(R+j X)\left(P_{m}-j Q_{m}\right) / V_{m}^{*} \\
V_{m}=V_{n}+\left(P_{m} \cdot R+Q_{m} \cdot X\right) / V_{m}^{*}+j\left(P_{m} \cdot X-Q_{m} \cdot R\right) / V_{m}^{*}
\end{gathered}
$$


Considering the diagram phasor in fig 4 :

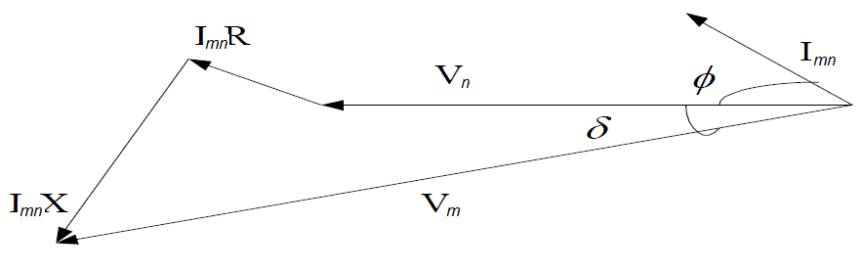

Figure 4. Phasor diagram

$$
V_{m} \cdot \sin \delta=\left(P_{m} \cdot X-Q_{n} \cdot R\right) / V_{n}
$$

Since the voltage angle $\delta$ is very small, the term $\left(P_{m} . X-Q_{n} \cdot R\right) / V_{n}$ can be neglected, and the equ (12) become:

$$
V_{m}=V_{n}+\left(P_{m} \cdot R+Q_{m} \cdot X\right) / V_{m}^{*}
$$

And the voltage difference between the nodes " $\mathrm{m}$ " and " $\mathrm{n}$ " can be expressed as:

$$
\Delta V=\left(P_{m} \cdot R+Q_{m} \cdot X\right) / V_{m}^{*}
$$

With the presence of a distributed generation unit in the node "m":

$$
P_{m}=P_{L}-P_{n} \quad \text { and } \quad Q_{m}=Q_{L} \pm Q_{n}
$$

So, the voltage difference between the nodes " $m$ " and " $n$ ", with the presence of a distributed generation unit in the node "m":

$$
\left.\Delta V=\left[\left(P_{L}-P_{n}\right) \cdot R+\left(Q_{L} \pm Q_{n}\right) \cdot X\right)\right] / V_{m}^{*}
$$

The active power produced by distributed generation unit increase the voltage, whereas the reactive power can further increase or reduce it depending on the type of DG.

These outcomes, in combination with the distribution network characteristics $(\mathrm{R} / \mathrm{X})$ and load profile, determine whether the voltage plan.

\section{Proposed Mathematic Analysis Method}

The voltage of a node " $x$ ", depends on the powers injected or absorbed at the other network nodes:

$$
V_{x}=V_{x}\left(P_{a}^{\circ}, P_{b}^{\circ}, \ldots, P_{m \circ}^{\circ}, Q_{a}^{\circ}, Q_{b}^{\circ}, \ldots, Q_{m \circ}^{\circ}\right)
$$

Changes in voltage are related to changes in active power $(\mathrm{P})$ and reactive power $(\mathrm{Q})$ by the partial differential equation:

$$
\left[\begin{array}{c}
\Delta V_{\circ} \\
\circ \\
\Delta \delta_{\circ}
\end{array}\right]=\left[\begin{array}{ccc}
\frac{\partial V_{\circ}}{\partial P_{\circ}} & \circ & \frac{\partial V_{n}}{\partial Q_{z}} \\
\circ & \circ & \circ \\
\frac{\partial \delta_{9}}{\partial Q_{z}} & \circ & \frac{\partial \delta_{+}}{\partial Q_{u}}
\end{array}\right] x\left[\begin{array}{c}
\Delta P_{\circ} \\
\circ \\
\Delta Q_{\circ}
\end{array}\right]
$$

Let's consider [F] a column vector of complex power of power nodes, the dimension of $[\mathrm{S}]$ is $(\mathrm{mxxx} 1)$ :

$$
[F]=\left[V_{\circ}^{\circ}\right] *\left[I^{*}{ }_{0}^{\circ}\right]=[P]+j[Q]
$$

Where $[\mathrm{P}]$ and $[\mathrm{Q}]$ are the column vectors, dimension $(\mathrm{m} \times 1)$, whose elements are, respectively, the node active and re- 
active powers.

The complex conjugate of $[\mathrm{S}]$ is given by:

$$
\left[F^{*}\right]=\left[V_{\circ}^{*}\right] *\left[I^{S_{\circ}^{0}}\right]=[P]-j[Q]
$$

The column vector of current at each node:

$$
[I]=\frac{[P]-j[Q]}{\left[V^{*}\right]}
$$

The current at each node is often called the current injections, and the current between two nodes is called the branch current, the relationship between the bus current injections and branch current can be obtained by applying Kirchhoff's current law. The branch currents can then be formulated as functions of equivalent current injections.

Let's consider the single line diagram in fig 5.

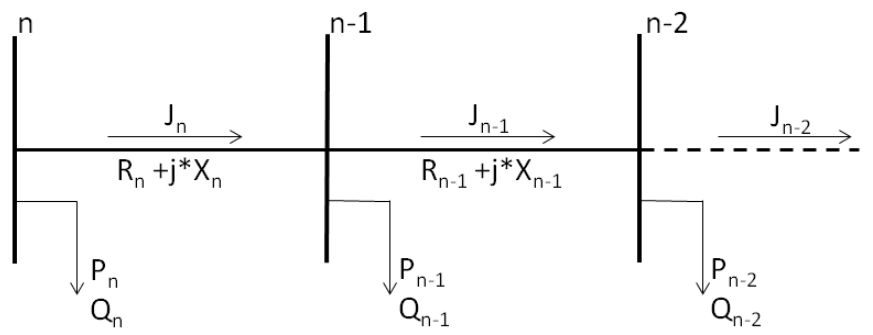

Figure 5. Single line diagram

The electric current between the terminal node " $n$ " and the next node " $n-1$ " is:

$$
J_{n}^{k}=\operatorname{conj}\left(\frac{P_{n}+j * Q_{n}}{V_{n}^{k}}\right)
$$

The electric current between a sending node (n-i) and a receiving node (n-i-1), by applying Kirchhoff's current law, the branch currents, can be expressed by equivalent current injections as:

$$
J_{n-i}^{k}=-\operatorname{conj}\left(\frac{P_{n-i}+j * Q_{n-i}}{V_{n-i}^{k}}\right)+\sum_{r} J_{n-r}^{k} \quad r=1 \ldots
$$

Where $\sum_{r} J_{n-r}^{k}$ is the currents in branches emanating from the node "n-i".

The relationship between the current of each node or the bus current injections and branch currents can be obtained from equ (16):

$$
[J]=[B I B C] \times[I]
$$

The constant Bus Injection to Bus Current "BIBC" matrix is an upper triangular matrix with two values of 0 and +1 .

The relationship between branch currents and bus voltages can be obtained using the computed current, an updating of voltages values start from the root bus, the node voltages are:

$$
V_{n}^{k}=V_{n-1}^{k}-\left(R_{n}+j * X_{n}\right) * J_{n}^{k}
$$

Therefore the relationship between branch currents and bus voltages can be expressed as:

$$
[\Delta V]=[B C B V] \times[J]
$$

The Bus Current to Bus Voltage "BCBV" can used to compute the variations at bus voltage generated by branch currents variations. 
The BIBC and BCBV matrices are generated based only on the topological structure of distribution systems. Combining equation (24) and (26), the relationship between bus current injections and bus voltages can be expressed as:

$$
[\Delta V]=[B C B V][B I B C][I]=[D L F][I]
$$

Where DLF is a multiplication matrix of BCBV and BIBC matrices

Let's consider two matrices $[\mathrm{R}]$ and $[\mathrm{X}]$ as:

$$
[\mathrm{R}]=\operatorname{real}([\mathrm{Z}]) \text { and }[\mathrm{X}]=\operatorname{im}([\mathrm{Z}])
$$

The elements $(\mathrm{x} ; \mathrm{y})$ of the matrix $[\mathrm{R}]$ are the sum of the impedance of the branches in which both $\mathrm{P}_{\mathrm{x}+1}$ and $\mathrm{P}_{\mathrm{y}+1}$ flow. For instance, in order to obtain the element $(3,2)$ of $[\mathrm{R}]$.

$$
[V]=\left[V_{\text {substation }}\right]-([R]+j[X]) *\left(\frac{[P]-j[Q]}{\left[V^{*}\right]}\right)
$$

Equ (29) can be simplified considering the hypotheses below (commonly accepted in distribution networks analysis) [5]:

- The phase difference between node voltages is negligible and, as a consequence, if phasor $\mathrm{V}_{1}$ is chosen on the real axis, only the real part of voltage $[\mathrm{V}]=$ real[ $[\mathrm{V}]$ is considered.

- Constant current models are considered for loads (node powers are referred to system nominal voltage instead of actual node voltage).

Equations (23) can be written as:

$$
[V]=\left[V_{\text {Substation }}\right]-([R]+j[X]) *\left(\frac{[P]-j[Q]}{V_{\text {nom }}}\right)
$$

For a node $\mathrm{x}$ :

$$
V_{i}=V_{0}-\frac{1}{V_{\text {nom }}}\left(\sum R_{i j} P_{j}+\sum X_{i j} Q_{j}\right)
$$

From equation (25), the voltage of node $\mathrm{x}$ depend on the active and reactive power injections or consumed of all node networks.

As the substation node is the slack bus, its voltage is always constant.

$$
\left\{\begin{array}{l}
\frac{\partial V_{x}}{\partial P_{y}}=-\frac{1}{V_{n o m}} R_{x y}=-\frac{1}{V_{n o m}} L_{x y} r_{x y} \\
\frac{\partial V_{x}}{\partial Q_{y}}=-\frac{1}{V_{n o m}} X_{x y}=-\frac{1}{V_{n o m}} L_{x y} x_{x y}
\end{array}\right.
$$

Where $\mathrm{L}_{\mathrm{xy}}$ are:

- for $x=y$ the sum of the branch lengths forming the path from the origin (node 0 ) to node $\mathrm{i}$

- for $\mathrm{x} \neq \mathrm{y}$ the sum of the branch lengths forming the path from the origin to the common node of the paths formed by the origin and nodes $i$ and $j$.

The total differential of function $\mathrm{V}_{\mathrm{x}}$ is:

Considering all network nodes:

$$
d V_{x}=\sum \frac{\partial V_{x}}{\partial P_{y}} d P_{y}+\sum \frac{\partial V_{x}}{\partial Q_{y}} d Q_{y}
$$




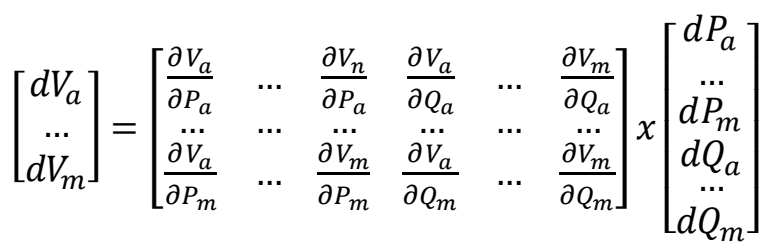

In the next section a case study is with the IEEE 69-bus radial distribution system.

\section{Case study}

As provided in section III, the voltage of a node " $\mathrm{x}$ ", depends on the powers injected or absorbed at the other network nodes:

$$
V_{x}=V_{x}\left(P_{a \circ}^{\circ}, P_{b \circ}^{\circ}, \ldots, P_{m \circ}^{\circ}, Q_{a \circ}^{\circ}, Q_{b \circ}^{\circ}, \ldots, Q_{m \circ}^{\circ}\right)
$$

The proposed mathematic method is tested in this section with a the $12.66 \mathrm{KV}$ network IEEE 69 bus, the single line diagram is shown in fig 4, the topology characteristics of the network is presented in table 1 [6].

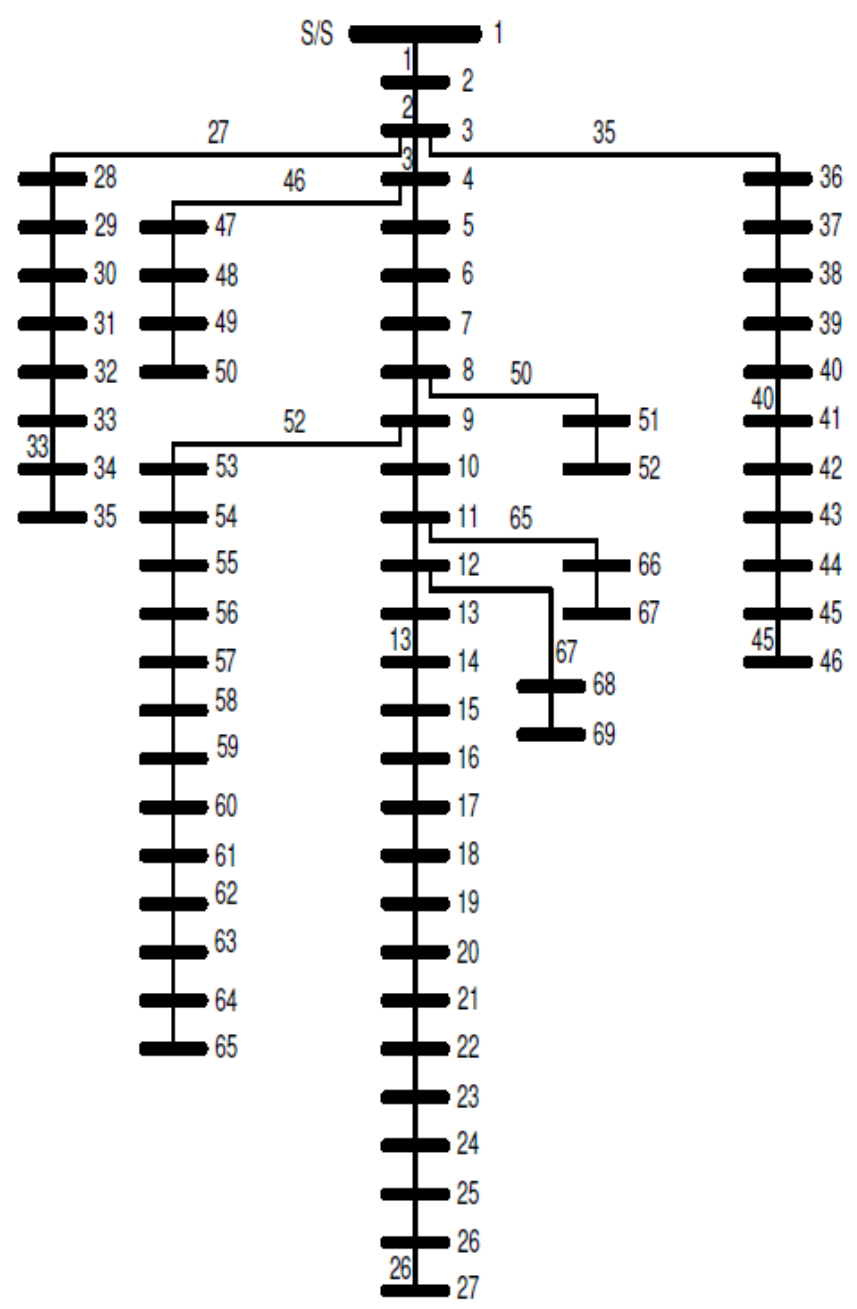

Figure 6. Single phase diagram of IEE 69-bus distribution system 
From a Line Data, that indicates the resistance and reactance of each branches, and the topological structure of the network, The BIBC and BCBV matrices are developed and the DLF matrix is calculated as the multiplication of the matrices $\mathrm{BIBC}$ and $\mathrm{BCBV}$.

The characteristics of each branch of IEEE 69-bus is presented in the table I, also the result of sensitivity coefficients are added on the same table, at columns 6 and 7.

From the sensitivity coefficients is to highlight that the most important values are recorded at nodes: $26 ; 34 ; 64 ; 67$ and 69, which represent the ends of the network branches and the most distance nodes from the substation. Further, it can be easily highlighted that the greater the node distances from the origin, had the higher the voltage sensitivity.

Table I. Line Data of the Medium voltage radial network

\begin{tabular}{|c|c|c|c|c|c|c|}
\hline $\begin{array}{l}\text { Branch } \\
\text { Num } \\
\text {-ber }\end{array}$ & $\begin{array}{l}\text { Send } \\
\text {-ing } \\
\text { Bus }\end{array}$ & $\begin{array}{l}\text { Recei } \\
\text {-ving } \\
\text { Bus }\end{array}$ & $\begin{array}{l}\text { Resist } \\
\text {-ance }\end{array}$ & $\begin{array}{l}\text { React } \\
\text {-ance }\end{array}$ & $\mathrm{Sp}$ & $\mathrm{Sq}$ \\
\hline 1 & 1 & 2 & 0.0005 & 0.0012 & 0.0000 & -0.0001 \\
\hline 2 & 2 & 3 & 0.0005 & 0.0012 & -0.0001 & -0.0002 \\
\hline 3 & 3 & 4 & 0.0015 & 0.0036 & -0.0002 & -0.0005 \\
\hline 4 & 4 & 5 & 0.0251 & 0.0294 & -0.0022 & -0.0028 \\
\hline 5 & 5 & 6 & 0.6330 & 0.1864 & -0.0522 & -0.0175 \\
\hline 6 & 6 & 7 & 0.3811 & 0.1941 & -0.0823 & -0.0329 \\
\hline 7 & 7 & 8 & 0.0922 & 0.0470 & -0.0896 & -0.0366 \\
\hline 8 & 8 & 9 & 0.0493 & 0.0251 & -0.0935 & -0.0385 \\
\hline 9 & 9 & 10 & 0.8190 & 0.2707 & -0.1582 & -0.0599 \\
\hline 10 & 10 & 11 & 0.1872 & 0.0619 & -0.1729 & -0.0648 \\
\hline 11 & 11 & 12 & 0.7114 & 0.2351 & -0.2291 & -0.0834 \\
\hline 12 & 12 & 13 & 1.0300 & 0.3400 & -0.3105 & -0.1102 \\
\hline 13 & 13 & 14 & 1.0440 & 0.3450 & -0.3930 & -0.1375 \\
\hline 14 & 14 & 15 & 1.0580 & 0.3496 & -0.4765 & -0.1651 \\
\hline 15 & 15 & 16 & 0.1966 & 0.0650 & -0.4921 & -0.1702 \\
\hline 16 & 16 & 17 & 0.3744 & 0.1238 & -0.5216 & -0.1800 \\
\hline 17 & 17 & 18 & 0.0047 & 0.0016 & -0.5220 & -0.1802 \\
\hline 18 & 18 & 19 & 0.3276 & 0.1083 & -0.5479 & -0.1887 \\
\hline 19 & 19 & 20 & 0.2106 & 0.0690 & -0.5645 & -0.1942 \\
\hline 20 & 20 & 21 & 0.3416 & 0.1129 & -0.5915 & -0.2031 \\
\hline 21 & 21 & 22 & 0.0140 & 0.0046 & -0.5926 & -0.2034 \\
\hline 22 & 22 & 23 & 0.1591 & 0.0526 & -0.6052 & -0.2076 \\
\hline 23 & 23 & 24 & 0.3463 & 0.1145 & -0.6325 & -0.2166 \\
\hline 24 & 24 & 25 & 0.7488 & 0.2475 & -0.6917 & -0.2362 \\
\hline 25 & 25 & 26 & 0.3089 & 0.1021 & -0.7161 & -0.2442 \\
\hline 26 & 26 & 27 & 0.1732 & 0.0572 & -0.7297 & -0.2488 \\
\hline 27 & 3 & 28 & 0.0044 & 0.0108 & -0.0004 & -0.0010 \\
\hline 28 & 28 & 29 & 0.0640 & 0.1565 & -0.0055 & -0.0134 \\
\hline 29 & 29 & 30 & 0.3978 & 0.1315 & -0.0369 & -0.0238 \\
\hline 30 & 30 & 31 & 0.0702 & 0.0232 & -0.0424 & -0.0256 \\
\hline 31 & 31 & 32 & 0.3510 & 0.1160 & -0.0702 & -0.0348 \\
\hline 32 & 32 & 33 & 0.8390 & 0.2816 & -0.1364 & -0.0570 \\
\hline 33 & 33 & 34 & 1.7080 & 0.5646 & -0.2714 & -0.1016 \\
\hline
\end{tabular}




\begin{tabular}{|l|l|l|l|l|l|l|}
34 & 34 & 35 & 1.4740 & 0.4873 & -0.3878 & -0.1401 \\
\hline 35 & 3 & 36 & 0.0044 & 0.0108 & -0.0004 & -0.0010 \\
\hline 36 & 36 & 37 & 0.0640 & 0.1565 & -0.0055 & -0.0134 \\
\hline 37 & 37 & 38 & 0.1053 & 0.1230 & -0.0138 & -0.0231 \\
\hline 38 & 38 & 39 & 0.0304 & 0.0355 & -0.0162 & -0.0259 \\
\hline 39 & 39 & 40 & 0.0018 & 0.0021 & -0.0163 & -0.0261 \\
\hline 40 & 40 & 41 & 0.7283 & 0.8509 & -0.0739 & -0.0933 \\
\hline 41 & 41 & 42 & 0.3100 & 0.3623 & -0.0984 & -0.1219 \\
\hline 42 & 42 & 43 & 0.0410 & 0.0478 & -0.1016 & -0.1257 \\
\hline 43 & 43 & 44 & 0.0092 & 0.0116 & -0.1023 & -0.1266 \\
\hline 44 & 44 & 45 & 0.1089 & 0.1373 & -0.1109 & -0.1375 \\
\hline 45 & 45 & 46 & 0.0009 & 0.0012 & -0.1110 & -0.1376 \\
\hline 46 & 4 & 47 & 0.0034 & 0.0084 & -0.0005 & -0.0011 \\
\hline 47 & 47 & 48 & 0.0851 & 0.2083 & -0.0072 & -0.0176 \\
\hline 48 & 48 & 46 & 0.2898 & 0.7091 & -0.0301 & -0.0736 \\
\hline 49 & 49 & 50 & 0.0822 & 0.2011 & -0.0366 & -0.0895 \\
\hline 50 & 8 & 51 & 0.0928 & 0.0473 & -0.0974 & -0.0403 \\
\hline 51 & 51 & 52 & 0.3319 & 0.1114 & -0.1236 & -0.0491 \\
\hline 52 & 52 & 53 & 0.1740 & 0.0886 & -0.1373 & -0.0561 \\
\hline 53 & 9 & 54 & 0.2030 & 0.1034 & -0.1095 & -0.0467 \\
\hline 54 & 54 & 55 & 0.2842 & 0.1447 & -0.1319 & -0.0581 \\
\hline 55 & 55 & 56 & 0.2813 & 0.1433 & -0.1542 & -0.0695 \\
\hline 56 & 56 & 57 & 1.5900 & 0.5337 & -0.2798 & -0.1116 \\
\hline 57 & 57 & 58 & 0.7837 & 0.2630 & -0.3417 & -0.1324 \\
\hline 58 & 58 & 59 & 0.3042 & 0.1006 & -0.3657 & -0.1403 \\
\hline 59 & 59 & 60 & 0.3861 & 0.1172 & -0.3962 & -0.1496 \\
\hline 60 & 60 & 61 & 0.5075 & 0.2585 & -0.4363 & -0.1700 \\
\hline 61 & 61 & 62 & 0.0974 & 0.0496 & 0.0000 & -0.0001 \\
\hline 62 & 62 & 63 & 0.1450 & 0.0738 & -0.0001 & -0.0002 \\
\hline 63 & 63 & 64 & 0.7105 & 0.3619 & -0.0002 & -0.0005 \\
\hline 64 & 64 & 65 & 1.0410 & 0.5302 & -0.0022 & -0.0028 \\
\hline 65 & 11 & 66 & 0.2012 & 0.0611 & -0.0522 & -0.0175 \\
\hline 66 & 66 & 67 & 0.0047 & 0.0014 & -0.0823 & -0.0329 \\
\hline 67 & 12 & 68 & 0.7394 & 0.2444 & -0.0896 & -0.0366 \\
\hline 68 & 68 & 69 & 0.0047 & 0.0016 & -0.0935 & -0.0385 \\
\hline
\end{tabular}

In fig 7, a graphical representation is presented, shows the voltage sensitivity of the network nodes due to the variation of all loads active power. From the fig 7, we can identify 8 curves, with decreasing values, which exactly correspond to the 8 branches of the IEEE 69-bus network: Principal branch from node 1 to 27 , the branch from node 3 to node 35 , the branch from node 3 to node 46 , the branch from the node 8 to the node 52, the branch from the node 9 to the node 65 , the branch from the node 11 to the node 67, and the branch from the node 12 to the node 69 .

In fig 8 , a graphical representation is given, showing the variation of voltage plan with respect to the variation of all loads reactive power. From the fig 8 , we can also identify 8 curves, with decreasing values, which exactly correspond to the 8 branches of the IEEE 69-bus network: Principal branch from node 1 to 27, the branch from node 3 to node 35, the branch from node 3 to node 46 , the branch from the node 8 to the node 52, the branch from the node 9 to the node 65 , the branch from the node 11 to the node 67, and the branch from the node 12 to the node 69 .

Comparing fig 7 and fig 8 we can observe that the IEEE 69-bus is more sensitive to the variation of active power than 
reactive power.

Fig 7 and Fig 8, gives an immediate perception on the voltage plan of the IEEE 69-bus network, such graphical representation is useful to get the most influencing parts of the network and to predict the most optimal placement of distributed generation, that minimize the impact of the variation of its active and reactive power, table II presents the best 10 optimal locations for distributed generation, with their maximum impact factory on the voltage plan of the IEEE 69-bus.

Fig 7 and Fig 8, present also important information to predict the most optimal placement for voltage control actuator. As the most impacted nodes are the busbars with the greater distances from the origin, table III resumes the best optimal placement for voltage control actuator, such as traditional generators, micro-turbines, bank capacity and D-STATCOM.

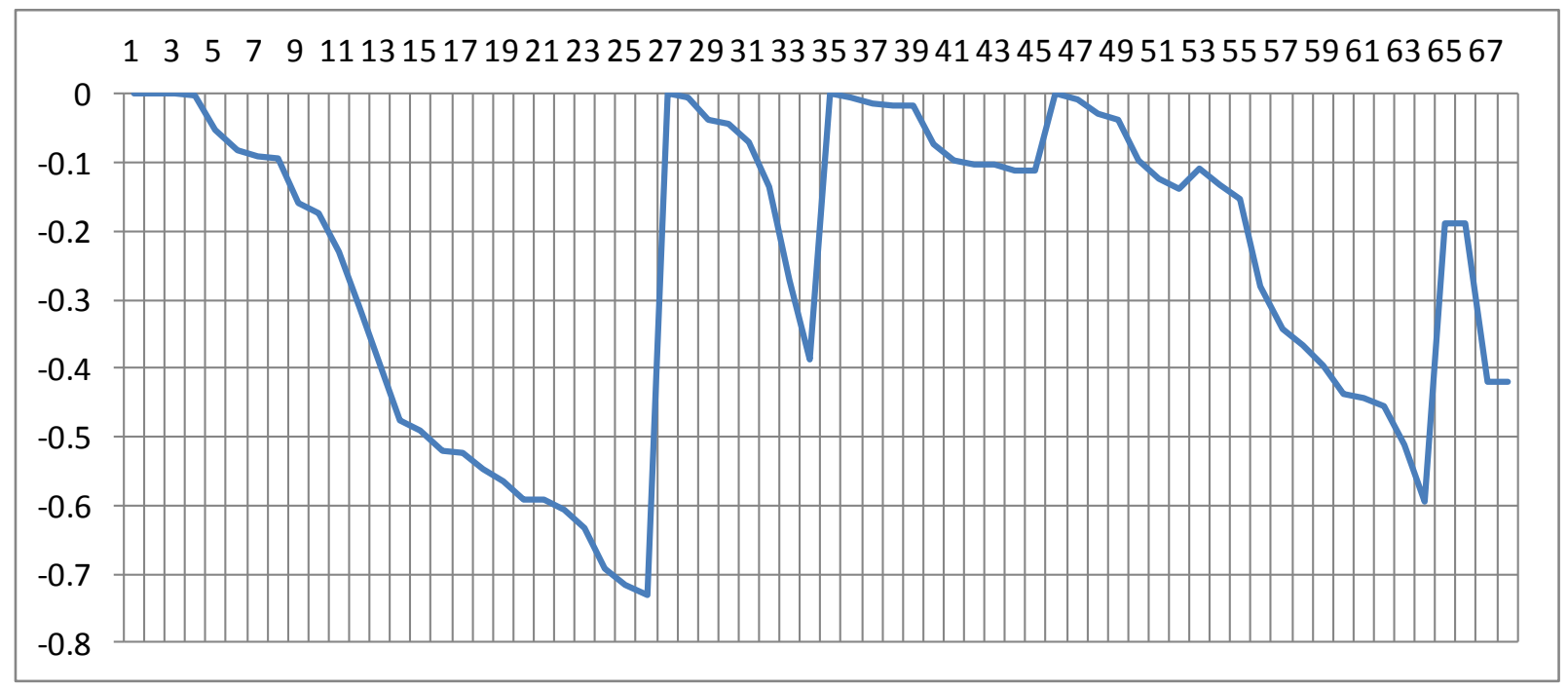

Figure 7. Voltage plan sensitivity with respect to the variation of all load active power

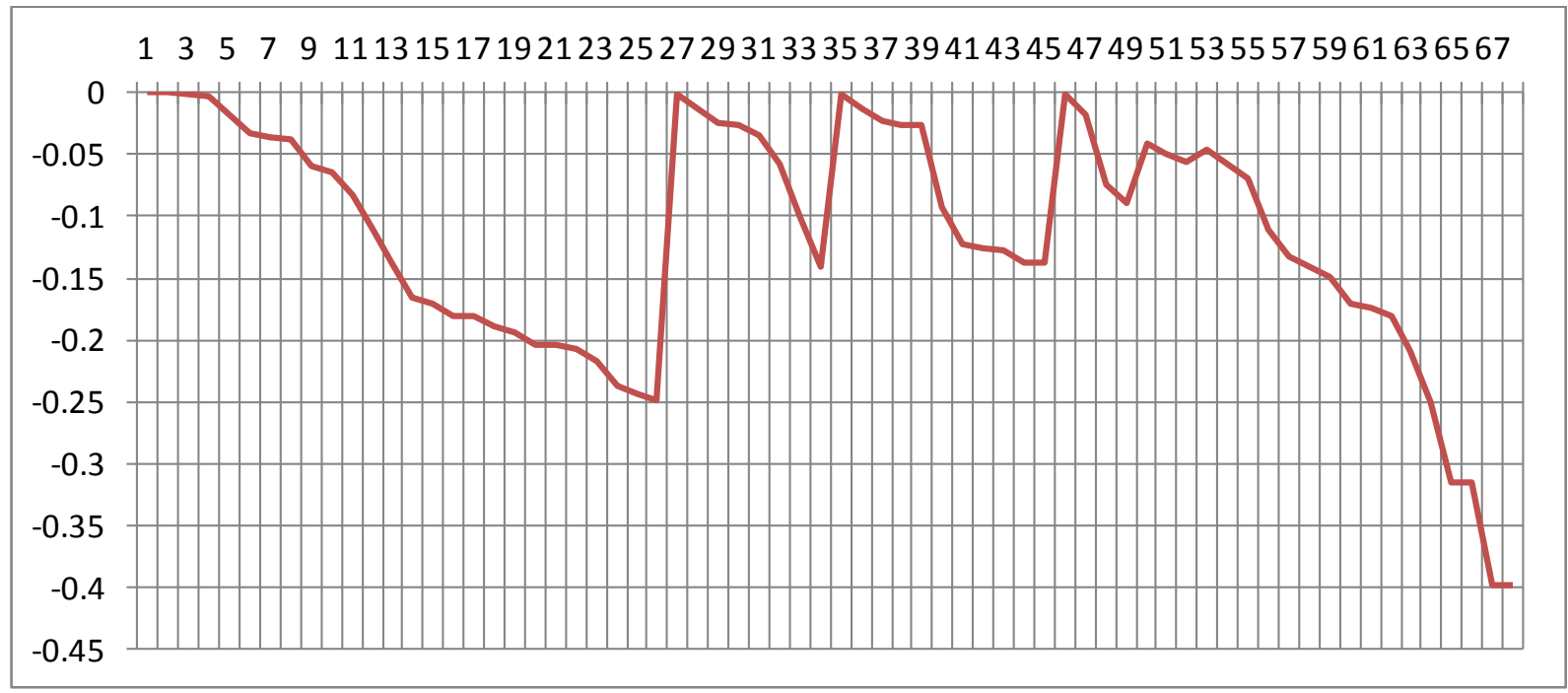

Figure 8 . Voltage plan sensitivity with respect to the variation of all load active power 
Table II. Optimal placement for distributed generation of the ieee 69-bus network

\begin{tabular}{|l|l|l|}
\hline $\begin{array}{l}\text { Node } \\
\text { Number }\end{array}$ & Fp & Fq \\
\hline 1 & $-0,0001$ & $-0,0001$ \\
\hline 2 & $-0,0001$ & $-0,0002$ \\
\hline 3 & $-0,0002$ & $-0,0005$ \\
\hline 3 & $-0,0004$ & $-0,0010$ \\
\hline 3 & $-0,0004$ & $-0,0010$ \\
\hline 4 & $-0,0005$ & $-0,0011$ \\
\hline 4 & $-0,0024$ & $-0,0035$ \\
\hline 28 & $-0,0075$ & $-0,0158$ \\
\hline 36 & $-0,0126$ & $-0,0282$ \\
\hline 47 & $-0,0193$ & $-0,0446$ \\
\hline
\end{tabular}

Table III. Optimal placement for voltage control actuator of the ieee 69-bus network

\begin{tabular}{|l|l|l|}
\hline $\begin{array}{l}\text { Node } \\
\text { Number }\end{array}$ & Fp & Fq \\
\hline 26 & $-0,7297$ & $-0,2488$ \\
\hline 25 & $-0,7161$ & $-0,2442$ \\
\hline 24 & $-0,6917$ & $-0,2362$ \\
\hline 23 & $-0,6325$ & $-0,2166$ \\
\hline 22 & $-0,6052$ & $-0,2076$ \\
\hline 64 & $-0,5938$ & $-0,2502$ \\
\hline 21 & $-0,5926$ & $-0,2034$ \\
\hline 20 & $-0,5915$ & $-0,2031$ \\
\hline 19 & $-0,5645$ & $-0,1942$ \\
\hline 18 & $-0,5479$ & $-0,1887$ \\
\hline
\end{tabular}

\section{Conclusions}

A analytical method to quantify the voltage sensitivity to the variation of active and reactive power variation is presented in this paper, The proposed method is developed based on the exploitation of the radial structure of medium voltage distribution system, and the backward/forward sweep concept, by forming two matrices: bus injection to bus current (BiBC) and branch current to bus voltage (BCBV).

As shown in this paper, the voltage of any node of the network depends on the active and reactive power of all network nodes. The proposed method is able to quantify this influence in a case of the variation of active or reactive power of any node of the network, on the voltage of any other part of the network.

The results obtained by the proposed method may be interesting for two applications:

- Choosing the connection point of a new DG, in the case of the presence of several feeders, in the vicinity of the DG site.

- Choosing the best way to control the voltage of a certain node of the network, by acting at the most influencing factor: active or reactive power and at which point.

\section{References}

[1] R. Shivarudraswamy, and D. N. Gaonkar: "Coordinated voltage control using multiple regulators in distribution system with distributed generators", World Academy of Science, Engineering and Technology, International Journal of Electrical, Computer, Energetic, Electronic and Communication Engineering Vol:5, No:2, 2011. 
[2] S. OUALI, A. Cherkaoui: "Load flow analysis for moroccan medium voltage distribution system", Conférence Internationale en Automatique \& Traitement de Signal (ATS-2018), Proceedings of Engineering and Technology - PET, Vol.36 pp.10-16.

[3] G. Mokhtari, A. Ghosh, G. Nourbakhsh, and G. Ledwich, "Smart robust resources control in LV network to deal with voltage rise issue," IEEE Trans. on Sustain. Energy, vol. 4, no. 4, pp. 1043-1050, Oct 2013.

[4] S. OUALI, A. Cherkaoui, "Sensitivity analysis in Medium voltage distribution systems," International journal of engineering research \& technology (IJERT), vol 7, issue 11, November-2018.

[5] S. CONTI, A.M. GRECO, S. RAITI: 'Voltage Sensitivity Analysis in MV Distribution Networks', the 6th WSEAS/IASME Int. Conf. on Electric Power Systems, High Voltages, Electric Machines, Tenerife, Spain, December 16-18, 2006.

[6] J. Moshtagh, S. Ghasemi , "Optimal Distribution System Reconfiguration Using Non-dominated Sorting Genetic Algorithm (NSGA-II)", Journal of Operation and Automation in Power Engineering, Vol. 1, No. 1, March 2013.

[7] W.El-Khattam, M.M.A Salama, "Distributed Generation Technologies, Defenitions and Benefits", Electric Power Systems Research 71 (2004) 119-128.

[8] A. Yadav, L. Srivastra, "Optimal Placement of Distributed Generation: An Overview and Key Issues", International conference on power, signals and computation (EPSCICON), 8-10 January 2014.

[9] S. Mishra, D. Das, "Load Flow Analysis for Unbalanced Radial Distribution Systems: A Comparison of Different Practical Methods", Journal of Electrical \& Electronics Engineering, Vol. II, No. 3, 2009.

[10]H.M. Mok and S.Elangovan M.M.A. Salama Dr Cao Longjian, "Power Flow Analysis for Balanced and Unbalanced Radial DistributionSystems" 